\title{
A Detective Story for Biomedical Footprints towards New Therapeutic Interventions in Diabetic Nephropathy
}

\author{
Toshio MiYatA and Kiyoshi KUROKAWA
}

\begin{abstract}
The biochemistry of protein modifications in human disease teaches us a number of lessons as it reveals factors and pathways implicated in the genesis of human pathology. For instance, diabetic renal damage is associated with a variety of stresses, e.g., glycemic stress resulting from hyperglycemia, oxidative stress from reactive oxygen species, carbonyl stress from reactive carbonyl compounds, and nitrosative stress from reactive nitrogen species. Proteins are particularly attractive targets for product analysis. Protein modifications are much more specific and stable biomarkers of the disease than lipids and other metabolites, and thus serve as footprints of biochemical processes. Their quantitative analysis not only facilitates better understanding of the physiopathology but also offers new therapeutic insight. In this review, we delineate oxidative protein modifications existing in diabetic nephropathy and discuss therapeutic perspectives towards the development of new classes of renoprotective agents.
\end{abstract}

(Internal Medicine 42: 1165-1171, 2003)

Key words: protein modification, advanced glycation end product, oxidative stress, carbonyl stress, renoprotection, anti-hypertensive agent

\section{Is There Oxidative Stress in Diabetes?}

In diabetes mellitus, the controversy has focused on the existence of an oxidative stress, its possible localization and relation to hyperglycemia, and its cause. Williamson et al (1) pointed out an increased cellular NADH/NAD ${ }^{+}$ratio and suggested that diabetes is a state of "reductive stress" and "pseudo-hypoxia" rather than "oxidative stress". The presence of oxidative stress in diabetes rests on indirect evidence including increased ratios of $\mathrm{NADP}^{+} / \mathrm{NADPH}$ and of oxidized to reduced glutathione, ascorbate, and albumin (2-4).
However, such imbalances in intracellular redox systems may be induced by non-oxidative mechanisms (e.g., the polyol pathway) and cause secondary alterations of sulfhydryl homeostasis, thus mimicking an oxidative stress (5). There is no a priori reason to conclude that these alterations necessarily indicate an oxidative stress.

The issue of oxidative stress in diabetes has been recently revisited by several researchers, who have relied upon new methodological approaches assessing protein modifications. The group of Baynes has first argued against the presence of a generalized oxidative stress in diabetes (6). These authors measure two oxidized amino acids in skin collagen, orthotyrosine and methionine sulfoxide. They demonstrated an age-dependent increase in oxidative damage in skin collagen, pointing out that aging is indeed an oxidative stressdependent phenomenon. Surprisingly, the age-adjusted levels of oxidized amino acids in collagen proved virtually identical in both diabetics and non-diabetics. They thus concluded that diabetes is not associated with a generalized oxidative stress.

On the other hand, we analyzed the protein modifications induced by carbohydrates and lipids occurring under oxidative stress and demonstrated the existence of a localized oxidative stress in diabetic nephropathy (7). Advanced glycation end products (AGEs) include a variety of molecular structures formed through different mechanisms. The formation of two well-known AGEs, pentosidine (8) and $N^{\varepsilon}$-(carboxymethyl)lysine (CML) (9), depends not only on glycation but also, to a greater extent, on oxidation (10). By contrast, pyrraline, another AGE, is formed only through a glucosedependent but oxidative stress-independent mechanism (11). Should tissue AGE formation depend only on hyperglycemia, all AGE structures should be detected in diabetic glomerular lesions. The results indicate that pentosidine and CML are identified in early mesangial expansion as well as in advanced nodular deposits, together with protein modifications derived from oxidation of lipids or amino acids (7, 12). By contrast, pyrraline is absent in diabetic glomeruli but readily identified in the interstitium. These findings implicate an oxidative stress rather than hyperglycemia in AGE

From Institute of Medical Sciences, Tokai University, Kanagawa

Reprint requests should be addressed to Dr. Toshio Miyata, Institute of Medical Sciences, Tokai University, 143 Shimokasuya, Isehara, Kanagawa 2591143 


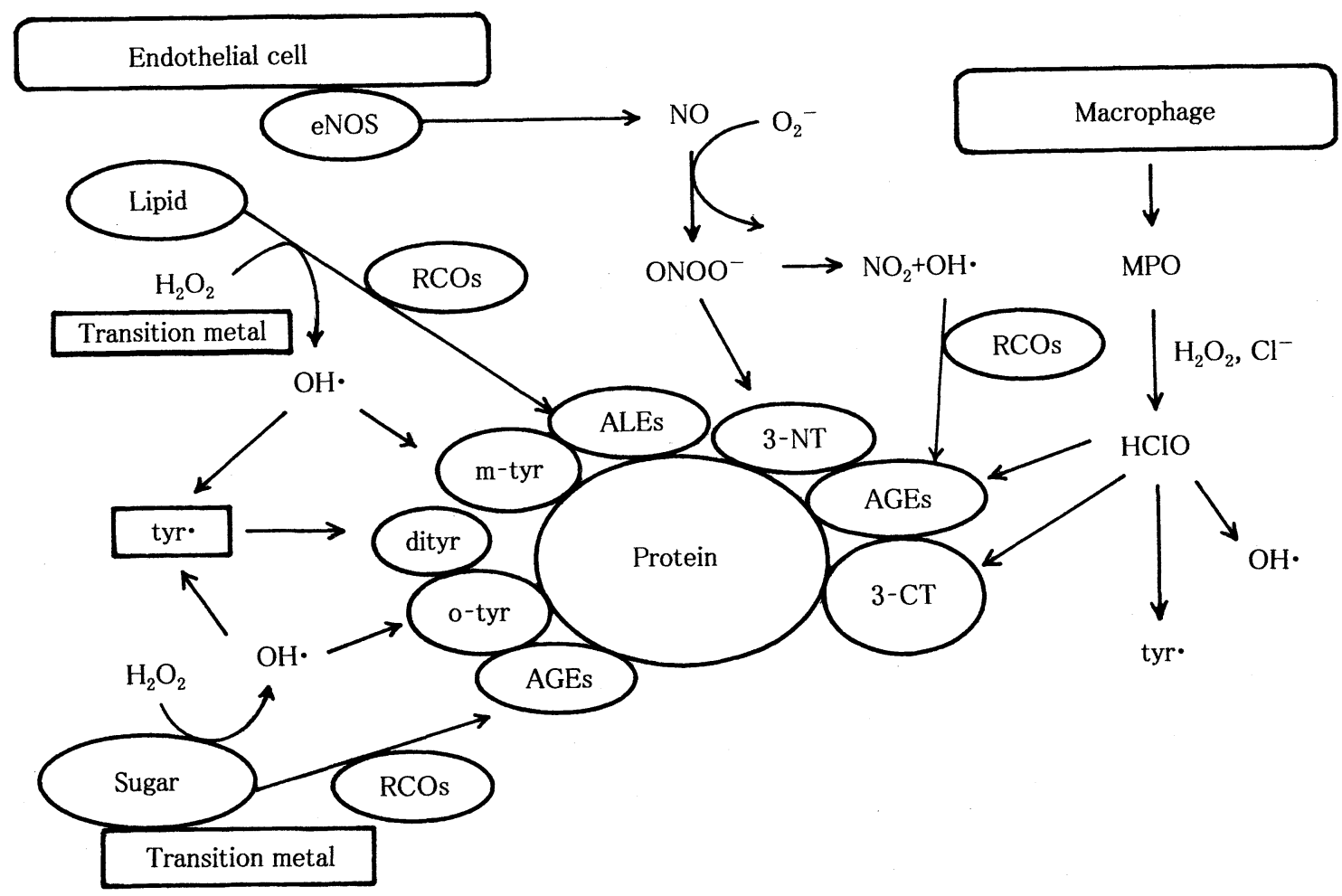

Figure 1. Oxidative protein damage in diabetic nephropathy.

formation of diabetic glomerular lesions.

The existence of a localized oxidative stress has been confirmed in diabetic vascular lesions by the group of Heinecke (13). In vascular lesions of streptozotocin-induced diabetic monkey, levels of ortho-tyrosine, meta-tyrosine, and dityrosine, all of which are protein modifications by hydroxyl and tyrosyl radicals, are significantly higher in diabetics than non-diabetics. Interestingly, these levels are correlated with hemoglobin A1c, suggesting a link between hyperglycemia and oxidative stress.

These findings altogether suggest that diabetes is not associated with a generalized oxidative stress, but with a localized oxidative stress (e.g., glomerular and vascular lesions).

\section{Various Oxidative Pathways and Their Protein Modifications}

In vivo, oxidative protein damage present in diabetic nephropathy is a complex phenomenon. It involves many factors and pathways as illustrated in Fig. 1. Hyperglycemiainduced glyco-oxidation generates some AGEs (e.g., pentosidine and CML). Reactive oxygen species, hydroxyl and tyrosyl radicals, produce ortho-tyrosine, meta-tyrosine, and di-tyrosine. Lipid peroxidation generates the advanced lipoxidation end products (ALEs), such as malondialdehydelysine, 4-hydroxynonenal adduct, and acrolein adduct $(5,14$, 15). Inflammatory cell-derived myeloperoxidase and hydrogen peroxide produce 3-chlorotyrosine (16). Nitric oxide reacts with superoxide and generates peroxynitrite, which produces 3-nitrotyrosine (17). Available evidence suggests that oxidative pathways differ between diabetic glomerular and vascular damage: an increase in 3nitrotyrosine is immunohistochemically detected in glomerular lesions of rats given streptozotocin (18) while it is absent by chemical analysis in vascular lesions of monkeys given streptozotocin (13). Identification of oxidative pathways actually implicated in human diabetic nephropathy is therefore of particular interest.

\section{Different Biochemical Profiles of Medical Drugs for Anti-oxidative Potential}

Once identified in humans, can these oxidative pathways be modified by therapeutic agents? Protein modifications may serve as useful markers and help ascertain the mechanism of action of the drug. Interestingly, several drugs currently used for human diseases interfere with oxidative protein damage through different mechanisms characteristic of their chemical structure.

For example, two well-known anti-hypertensive agents, nifedipine (a calcium channel blocker) and olmesartan (an angiotensin receptor blocker, ARB), share an antioxidative potential but their mechanism of action is different. Olmesartan is a potent scavenger for hydroxyl and tyrosyl 


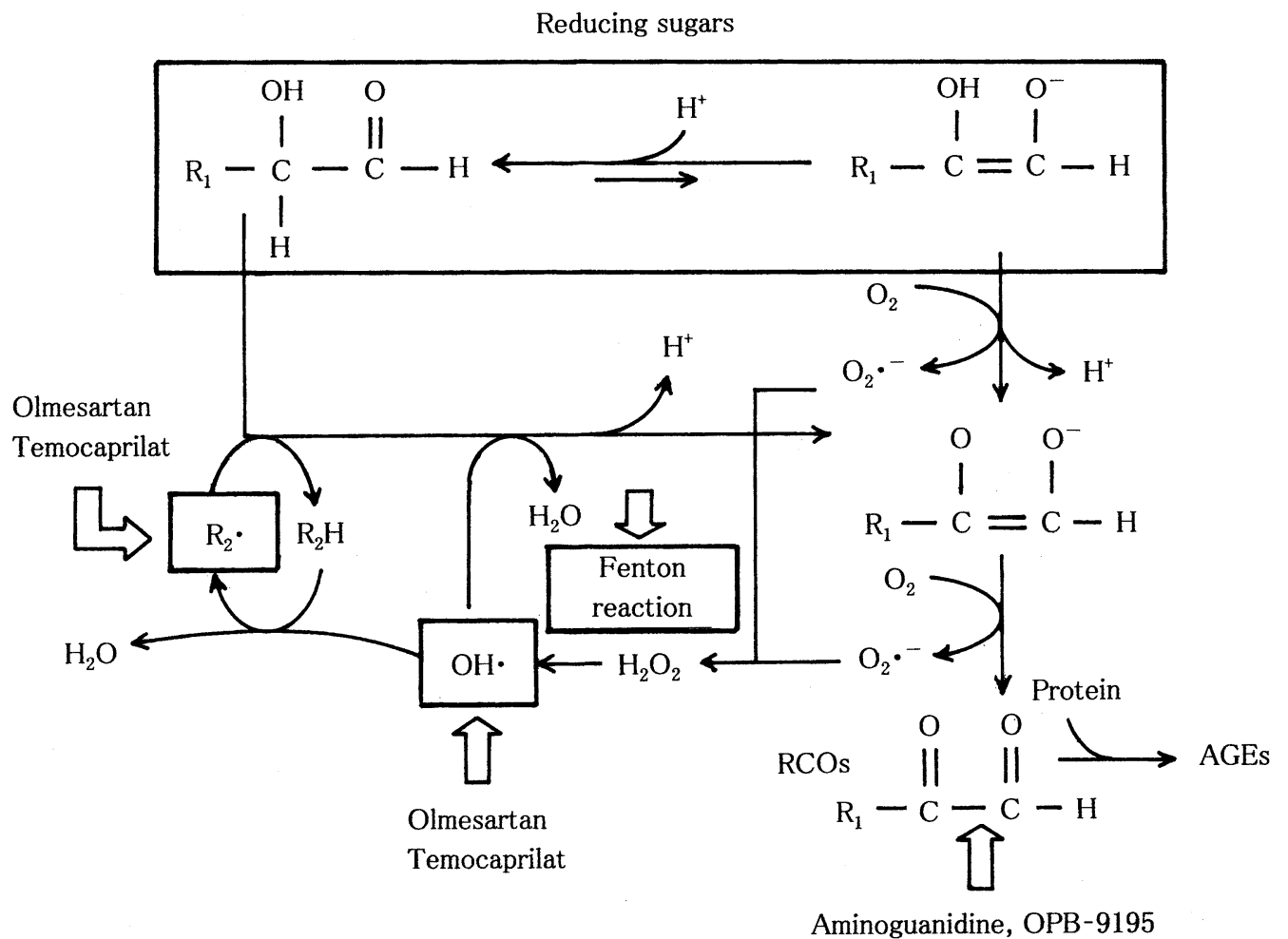

Figure 2. Inhibitory mechanisms of advanced glycation end products.

radicals and an inhibitor for both glyco-oxidation and lipoxidation reactions. Olmesartan thus inhibits in vitro the formation of AGEs, ALEs, ortho-tyrosine, meta-tyrosine, and di-tyrosine (our unpublished observations). Nifedipine, on the other hand, does not scavenge reactive oxygen species, but is a potent scavenger for peroxynitrite and inhibits in vitro the formation of 3-nitrotyrosine. Olmesartan and nifedipine thus act at different steps of oxidative protein damage. By contrast, atenolol (a beta-blocker) has little antioxidative potential.

Whether these in vitro findings can be extrapolated to in vivo models is as yet unknown. Still, these findings already open exciting prospects for new therapeutic interventions. They may also provide a rationale to utilize drugs according to their biochemical profile and the type of oxidative protein damage to be corrected in the microenvironment of disease.

\section{Therapeutic Perspectives: Inhibitors of AGEs, Carbonyl Stress, and Oxidative Stress}

The development of effective drugs that ameliorate the diabetic tissue damage has become a major therapeutic goal for several pharmaceutical companies. Among them is an inhibitor for the glyco-oxidation reaction (the so-called AGE or carbonyl stress inhibitor).

\section{The first generation AGE inhibitors}

Aminoguanidine is the first AGE inhibitor discovered in 1986 (19). This agent was followed by a more effective compound, ( \pm )-2-isopropylidenehydrazono-4-oxo-thiazolidin-5ylacetanilide (OPB-9195) (20). Both are hydrazinederivatives and inhibit in vitro the formation of AGEs, pentosidine and CML, from a variety of individual precursors including ribose, glucose and ascorbate, as well as that of two ALE moieties, malondialdehyde-lysine and 4hydroxynonenal-protein adduct, from arachidonate (21). They also inhibit pentosidine generation from diabetic and uremic plasma (22) or from conventional glucose based peritoneal dialysis fluid fortified with bovine serum albumin (23).

The mechanism of this inhibitory effect on AGE formation is complex. The hydrazine nitrogen atom of OPB-9195 reacts with reactive carbonyl compound ( $\mathrm{RCO}$ ) precursors for AGEs, directly or via the free base upon hydrolysis, to eventually form hydrazone (21). This mechanism is similar to that proposed for aminoguanidine.

OPB-9195 corrects several biological effects associated with AGE formation. In murine thymocyte and fibroblast cultures, it inhibits the phosphorylation of tyrosine residues of a number of intracellular proteins induced by cell surface Schiff base formation (24). In the experimental diabetic animal, such as the Otsuka-Long-Evans-Tokushima-Fatty (OLETF) rat, it reduces urinary albumin excretion and 
MIYATA and KUROKAWA
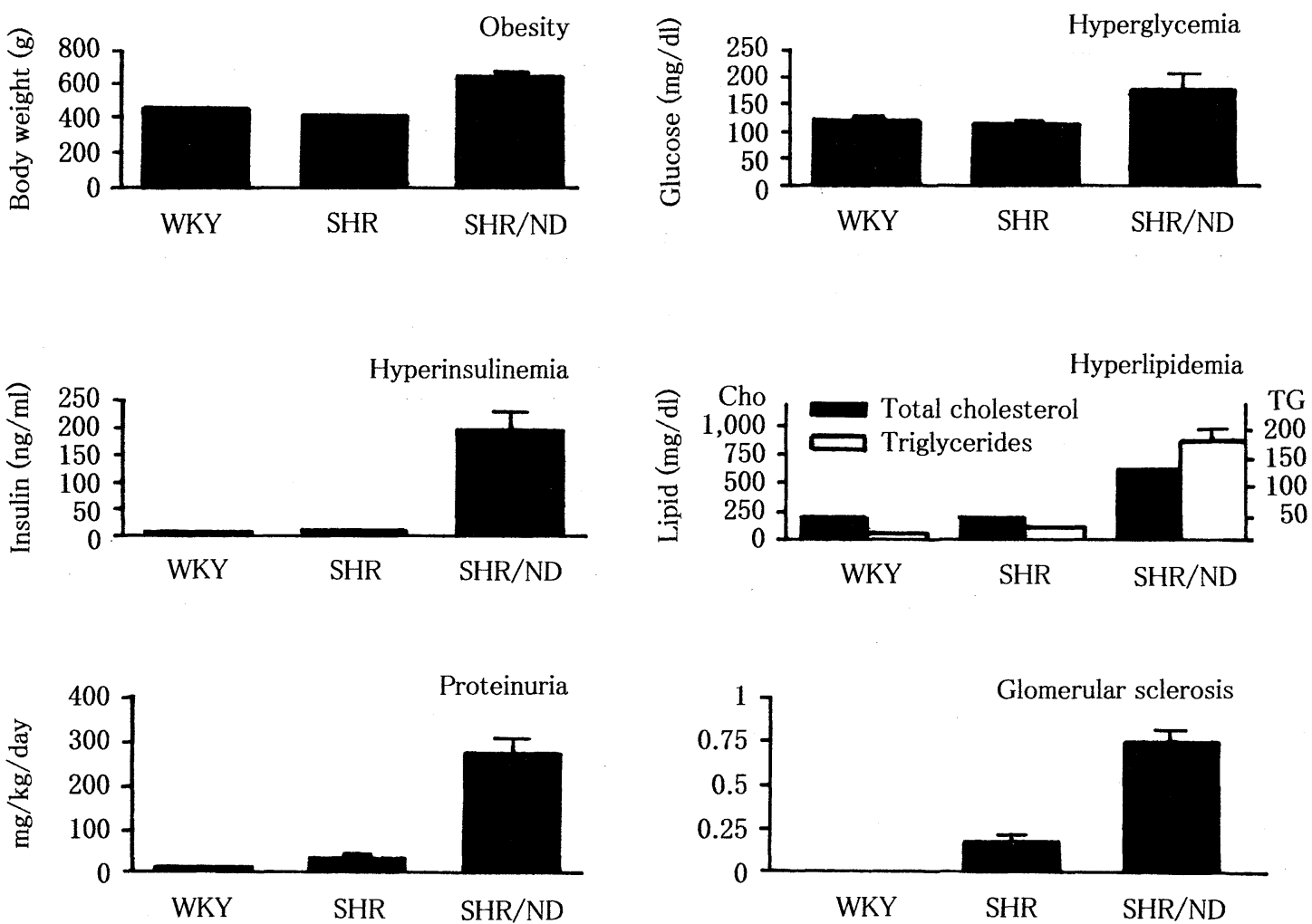

Figure 3. SHR/NDmc-cp (fat/fat) as a rat model of hypertensive type 2 diabetes and nephropathy. This figure is prepared from our data (J Am Soc Nephrol 14: 1212-1222, 2003).

improves glomerular morphology (20). Given to rats, after balloon injury of their carotid arteries, it effectively reduces neointima proliferation in arterial walls (25).

Unfortunately, the clinical benefits of this compound given to diabetic patients have been hampered by side effects, e.g., related to the characteristic trapping of pyridoxal with an attendant vitamin $B_{6}$ deficiency (26).

\section{Angiotensin II receptor blockers and angiotensin con- verting enzyme inhibitors}

We have therefore investigated the AGE inhibitory potential of other drugs whose tolerance had been previously demonstrated in clinical conditions. Unexpectedly, we found that angiotensin II receptor blockers (ARB) and angiotensin converting enzyme inhibitors (ACEI) lower the formation of pentosidine and CML (27). Inhibition is more striking for olmesartan (ARB) and temocaprilat (ACEI) than for aminoguanidine at the tested concentrations. The effect on AGE formation is common to 6 tested ARBs and their common core structure, 5-(4'-methylbiphenyl-2-yl)-1H-tetrazol. A similar but milder effect on AGE formation is observed with 4 ACEI tested at similar concentrations. However, no common core structure was identified in the tested ACEI.

The mechanism of the AGE lowering effect of ARB/ACEI differs markedly from that of aminoguanidine and OPB-9195 (27). Neither ARB nor ACEI entrap and lower in vitro the concentration of RCOs as well as of pyridoxal, in contrast to the first generation AGE inhibitors. Alternatively, ARB and ACEI decrease RCO production by interfering with several oxidative steps (Fig. 2), i.e., the formation of carbon-centered radicals and hydroxyl radicals, a characteristic shared only to a minor extent by aminoguanidine. ARB and ACEI further chelate transition metals implicated in the acceleration of AGE formation.

\section{AGE inhibition and renoprotection}

We have evaluated in vivo the AGE inhibitory effect of ARBs, in a unique model, the SHR/NDmc-cp (fat/fat) rat (28). This model (29) has the same genetic background as spontaneously hypertensive rats (SHR) and their original strain, Wistar-Kyoto rat (WKY). Like SHR, SHR/NDmc-cp (fat/fat) rats become hypertensive. Systemic blood pressure is much lower in SHR/NDmc-cp (fat/fat) $(170 \pm 9 \mathrm{mmHg}$ at 33 weeks) than in SHR rats $(192 \pm 5 \mathrm{mmHg}$ vs. $135 \pm 4 \mathrm{mmHg}$ in WKY). In the SHR/NDmc-cp (fat/fat) rats, hypertension is accompanied by a unique metabolic derangement (Fig. 3). In SHR, metabolic markers such as body weight and plasma levels of glucose, insulin, and lipids, are normal. In SHR/NDmc-cp (fat/fat) rats, by contrast, the genetic mutation of the leptin receptor (30) generates obesity $(650 \pm 14 \mathrm{~g}$ at 33 weeks vs. $417 \pm 6 \mathrm{~g}$ in SHR), hyperglycemia $(173 \pm 29$ $\mathrm{mg} / \mathrm{dl}$ vs. $113 \pm 2 \mathrm{mg} / \mathrm{dl}$ in SHR), hyperinsulinemia $(196 \pm 32$ 
$\mathrm{ng} / \mathrm{dl}$ vs. $6.0 \pm 0.6 \mathrm{ng} / \mathrm{dl}$ in SHR), and hyperlipidemia (triglyceride, $872 \pm 130 \mathrm{mg} / \mathrm{dl}$ vs. $102 \pm 8 \mathrm{mg} / \mathrm{dl}$ in SHR; total cholesterol, $128 \pm 6 \mathrm{mg} / \mathrm{dl}$ vs. $55 \pm 1 \mathrm{mg} / \mathrm{dl}$ in SHR), all characteristics of human type 2 diabetes.

The role of metabolic derangement in diabetic renal damage can be assessed independently of blood pressure levels by comparing SHR/NDmc-cp (fat/fat) with SHR rats. Despite severe hypertension, SHR has only a slight increase in proteinuria $(37.9 \pm 2.0 \mathrm{mg} / \mathrm{kg} / \mathrm{day}$ at 33 weeks vs. $15.0 \pm 1.4$ $\mathrm{mg} / \mathrm{kg} /$ day in WKY) and mild glomerular sclerosis. In SHR/NDmc-cp (fat/fat), by contrast, although hypertension is less severe, proteinuria $(276.2 \pm 31.2 \mathrm{mg} / \mathrm{kg} /$ day $)$ and glomerular sclerosis are much more severe (Fig. 3). In agreement with our common clinical experience, these observations point to the critical role played by metabolic derangements in diabetic renal damage.

Are these renal disorders preventable? Olmesartan $(5 \mathrm{mg} /$ $\mathrm{kg} /$ body weight/day) given to SHR/NDmc-cp (fat/fat) does not modify body weight or plasma levels of glucose and insulin, but decreases mildly plasma levels of triglyceride $(533 \pm 74 \mathrm{mg} / \mathrm{dl}$ at 33 weeks) and total cholesterol $(102 \pm 3$ $\mathrm{mg} / \mathrm{dl})$. Nevertheless, it reduces in a dose-dependent fashion systolic blood pressure $(118 \pm 6 \mathrm{mmHg})$ and diabetic nephropathy as evidenced by a decrease in proteinuria $(117.9 \pm 26.5 \mathrm{mg} / \mathrm{kg} /$ day $)$ and in pathologic evidence of glomerulosclerosis.

An impact on AGE genesis appears to be the key factor of the success of olmesartan. The pentosidine content in the kidney is similar in SHR $(0.040 \pm 0.003 \mathrm{pmol} / \mathrm{mg}$ of tissue proteins at 33 weeks) and WKY rats $(0.042 \pm 0.004 \mathrm{pmol} /$ $\mathrm{mg})$. Clearly, hypertension does not enhance AGE genesis. By contrast, the renal pentosidine content $(0.101 \pm 0.009$ $\mathrm{pmol} / \mathrm{mg}$ ) is markedly elevated in SHR/NDmc-cp (fat/fat). Impressively, administration of two different doses of olmesartan ( 1 and $5 \mathrm{mg} / \mathrm{kg} /$ body weight/day) to the latter rats reduces the renal pentosidine content in a dose-dependent way $(0.089 \pm 0.015 \mathrm{pmol} / \mathrm{mg}$ and $0.058 \pm 0.006 \mathrm{pmol} / \mathrm{mg}$, respectively). Of note, the renal pentosidine content proves significant correlation with proteinuria (28), supporting the existence of a link between AGE formation and renal damage.

Despite severe hypertension, non-diabetic SHR rats exhibit only a slight increase of proteinuria and glomerular damage (Fig. 3). In SHR/NDmc-cp (fat/fat), by contrast, proteinuria and glomerular sclerosis become much more severe. These findings points to the critical role played by sustained hyperglycemia with high insulin levels and/or obesity related hypercholesterolemia in the genesis of the marked renal lesions observed in SHR/ND mc-cp (fat/fat). The attendant advanced glycation of proteins, identified here by a high renal pentosidine content, might be a key element of the enhanced susceptibility of the diabetic kidney.

The additional, renoprotective benefits of ACEI or ARBs have been attributed to their ability to interfere with the renin-angiotensin system (RAS). To test this hypothesis, the efficacy of olmesartan has been compared with that of two other anti-hypertensive agents, acting independently of the

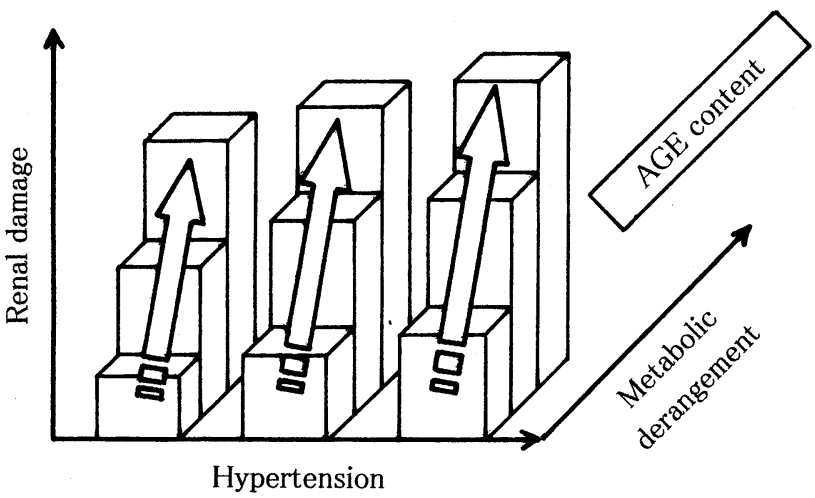

Figure 4. Association among hypertension, metabolic derangement (obesity, hyperglycemia, hyperinsulinemia, and hyperlipidemia), and diabetic renal damage.

RAS, hydralazine and atenolol. Hydralazine reduces in vitro pentosidine and CML production by trapping RCOs, just as aminoguanidine and OPB-9195, as well as by scavenging reactive oxygen species and chelating transition metals accelerating the Fenton reaction just as ACEI and ARB (28). By contrast, atenolol has no AG̣E inhibitory action (our unpublished observation).

Olmesartan and atenolol given to SHR/ND mc-cp (fat/fat) normalize systolic blood pressure to the same extent. However, only olmesartan reduces AGE renal content and successfully decreases proteinuria. Atenolol, by contrast, modifies neither renal AGE content nor proteinuria. In this model at least, these findings fit with the concept that some renal benefits of ARB are independent of blood pressure lowering but closely linked to AGE reduction (Table 1).

Contrary to atenolol, hydralazine significantly reduces the renal tissue pentosidine content and improves diabetic renal damage (28). The effect of hydralazine ( $5 \mathrm{mg} / \mathrm{kg} /$ day $)$ on albuminuria and glomerular damage, although not statistically significant, is similar to that observed with the low olmesartan dosage ( $1 \mathrm{mg} / \mathrm{kg} /$ day $)$, the blood pressure reduction being similar in the 2 groups. Renal protection appears thus independent of any interference with the RAS. Altogher, these results suggest that the added benefits of ARB in clinical trials may not be necessarily related to their ability to interfere with the RAS but might rather reflect an inhibition of AGE formation.

These considerations provide a framework to understand the additional benefits described in clinical studies of ARB in type 2 diabetics $(31,32)$. This hypothesis fits with the renoprotection afforded by AGE inhibitors in diabetic rats without hypertension $(20,33,34)$ or by ACEI and ARB in normotensive human diabetics (35).

The molecular mechanisms of the ARB induced renoprotection remain elusive. They may include improved endothelial cell function, probably associated with a decreased localized oxidative stress and AGE genesis. This hypothesis fits with clinical observations on the cardiovascular 
MIYATA and KUROKAWA

Table 1. Effects of Anti-hypertensive Agents or AGE Inhibitors on Blood Pressure Lowering, AGE Inhibition, and Renoprotection

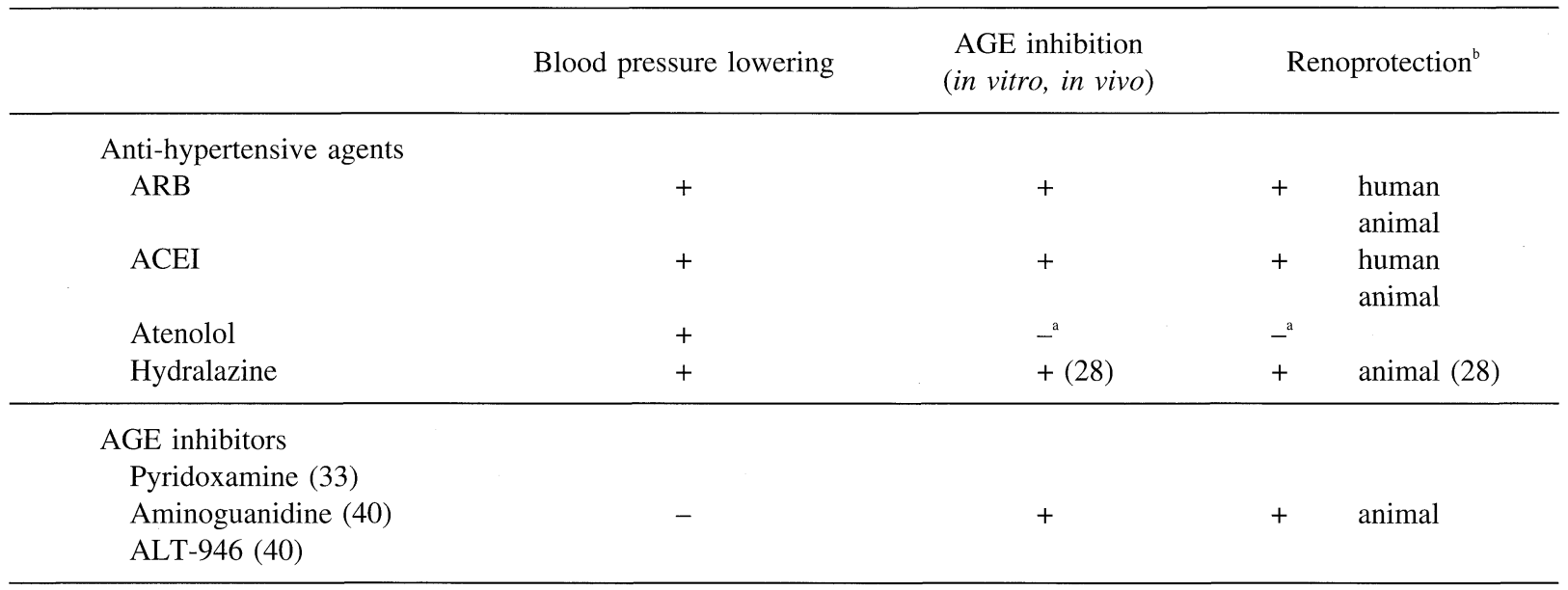

ARB: angiotensin II receptor blocker, ACEI: angiotensin-converting enzyme inhibitor, a: our unpublished observation, b: a decrease in proteinuria and in pathologic evidence of glomerulosclerosis.

and cerebrovascular protective actions of $\mathrm{ARB}(36,37)$. An interaction between oxidative stress, AGE formation, endothelial cell function, tissue injury or protection provides a new framework to better understand the systemic effects of ARB.

The discovery that renal AGE reduction correlates well with proteinuria reduction, raises prospects for the development of new classes of renoprotective agent. Some additional clinical and experimental data indeed support the hypothesis that renal protection may be obtained independently of blood pressure lowering. First, Weinberg et al (38) have administered candesartan, another ARB, at supramaximal doses up to $96 \mathrm{mg}$, to subjects with heavy proteinuria. Their preliminary data suggest that these high doses further reduce proteinuria without concomitant additional anti-hypertensive effect and despite complete angiotensin receptor blockade. The chemical effect rather than the biological action of ARB on angiotensin II, seems instrumental in lowering proteinuria.

Furthermore, we have observed in angiotensin II receptor type 1 knockout mice (kindly provided from Dr. Fukamidzu A, Center for Tsukuba Advanced Research Alliance, Institute of Applied Biochemistry) that oral administration of olmesartan effectively reduces neointima proliferation in carotid arteries after balloon injury (Dr. M. Sada, The University of Tokyo, School of Medicine, and T. Miyata, unpublished observation). This protective action is associated with tissue AGE reduction, without angiotensin II inhibition and blood pressure lowering effects.

Further evidence in favor of our interpretation has been recently reported in rats with streptozotocin-induced diabetes (Table 1). The group of Baynes (33) demonstrated that another AGE inhibitor, pyridoxamine, reduces AGE formation and contributes to renoprotection. Forbes et al (39) report that ramipril (ACEI) and aminoguanidine reduce to the same extent AGE accumulation in the kidney as measured by immunohistochemistry and fluorospectorometry. Finally, Wilkinson-Berka et al (40) observed that aminoguanidine as well as another AGE inhibitor, ALT-946, decreases AGE immunolabeling and provides renal protection.

\section{Future Prospects}

We have thus far characterized the AGE lowering profile of several thousands of chemical compounds including currently used medical drugs. Three classes of novel structures (not hydrazine-derivatives like aminoguanidine or ALT-946) have been identified that efficiently inhibit in vitro AGE formation: they are much more powerful and structurally different from the first generation AGE inhibitors, and do not trap pyridoxal. Several promising hit/lead compounds are now under investigation. Some of them decrease proteinuria efficiently in SHR/NDmc-cp without concomitant blood pressure and glucose lowering. We still have to synthesize their derivatives, optimize their structures, and test their effectiveness in preclinical studies. The development of these new drugs raises significant hopes to prevent or at least to slow down the development of diabetic nephropathy.

\section{References}

1) Williamson JR, Chang K, Frangos M, et al. Hyperglycemic pseudohypoxia and diabetic complications. Diabetes 42: 801-813, 1993.

2) Bravi MC, Pietrangeli $P$, Laurenti $O$, et al. Polyol pathway activation and glutathione redox status in non-insulin-dependent diabetic patients. Metabolism 46: 1194-1198, 1997.

3) Jennings PE, Chirico S, Jones AF, Lunec J, Barnett AH. Vitamin C metabolites and microangiopathy in diabetes mellitus. Diabetes Res 6 : 151-154, 1987.

4) Suzuki E, Yasuda K, Takeda N, et al. Increased oxidized form of human serum albumin in patients with diabetes mellitus. Diabetes Res 
Clin Pract 18:153-158, 1992.

5) Miyata T, van Ypersele de Strihou C, Kurokawa K, Baynes JW. Alterations in nonenzymatic biochemistry in uremia: origin and significance of "carbonyl stress" in long-term uremic complications. Kidney Int 55: 389-399, 1999.

6) Wells-Knecht MC, Lyons TJ, McCance DR, Thorpe SR, Baynes JW. Age-dependent increase in ortho-tyrosine and methionine sulfoxide in human skin collagen is not accelerated in diabetes. Evidence against a generalized increase in oxidative stress in diabetes. J Clin Invest 100: 839-846, 1997, 100.

7) Horie K, Miyata $T$, Maeda $K$, et al. Immunohistochemical colocalization of glycoxidation products and lipid peroxidation products in diabetic renal glomerular lesions. J Clin Invest 100: 29953004, 1997.

8) Sell DR, Monnier VM. Structure elucidation of a senescence cross-link from human extracellular matrix. J Biol Chem 264: 21597-21602, 1989.

9) Ahmed MU, Thorpe SR, Baynes JW. Identification of $N^{e}$-carboxymethyllysine as a degradation product of fructoselysine in glycated protein. J Biol Chem 261: 4889-4894, 1986.

10) Miyata $T$, Maeda $K$, Kurokawa $K$, van Ypersele de Strihou $C$. Oxidation conspires with glycation to generate noxious advanced glycation end products in renal failure. Nephrol Dial Transplant 12: 255-258, 1997.

11) Miyata S, Monnier VM. Immunohistochemical detection of advanced glycosylation end products in diabetic tissues using monoclonal antibody to pyrraline. J Clin Invest 89: 1102-1112, 1992.

12) Suzuki D, Miyata T, Saotome N, et al. Immunohistochemical evidence for an increased oxidative stress and carbonyl modification of proteins in diabetic glomerular lesions. J Am Soc Nephrol 10: 822-832,1999.

13) Pennathur S, Wagner JD, Leeuwenburgh C, Litwak KN, Heinecke JW. A hydroxyl radical-like species oxidizes cynomolgus monkey artery wall proteins in early diabetic vascular disease. J Clin Invest 107: 853$860,2001$.

14) Requena JR, Fu MX, Ahmed MU, et al. Quantification of malondialdehyde and 4-hydroxynonenal adducts to lysine residues in native and oxidized human low-density lipoprotein. Biochem J 322: $317-$ 325, 1997.

15) Uchida K, Kanematsu M, Sakai K, et al. Protein-bound acrolein: potential markers for oxidative stress. Proc Natl Acad Sci USA 95: 48824887, 1998.

16) Hazen SL, Hsu FF, Mueller DM, Crowley JR, Heinecke JW. Human neutrophils employ chlorine gas as an oxidant during phagocytosis. J Clin Invest 98: 1283-1289, 1996.

17) Ischiropoulos $\mathrm{H}$, al-Mehdi $\mathrm{AB}$. Peroxynitrite-mediated oxidative protein modifications. FEBS Lett 364: 279-282, 1995.

18) Onozato ML, Tojo A, Goto A, Fujita T, Wilcox CS. Oxidative stress and nitric oxide synthase in rat diabetic nephropathy: effects of ACEI and ARB. Kidney Int 61: 186-194, 2002.

19) Brownlee M, Vlassara H, Kooney A, Ulrich P, Cerami A. Aminoguanidine prevents diabetes-induced arterial wall protein crosslinking. Science 232: 1629-1632, 1986.

20) Nakamura $S$, Makita $Z$, Ishikawa $S$, et al. Progression of nephropathy in spontaneous diabetic rats is prevented by OPB-9195, a novel inhibitor of advanced glycation. Diabetes 46: 895-899, 1997.

21) Miyata $T$, Ueda $Y$, Asahi $K$, et al. Mechanism of the inhibitory effect of OPB-9195 [( \pm )-2-isopropylidenehydrazono-4-oxo-thiazolidin-5ylacetanilide] on advanced glycation end product and advanced lipoxidation end product formation. J Am Soc Nephrol 11: 1719$1725,2000$.

22) Miyata T, Ueda $Y$, Yamada $Y$, et al. Accumulation of carbonyls accelerates the formation of pentosidine, an advanced glycation end product: carbonyl stress in uremia. J Am Soc Nephrol 9: 2349-2356, 1998.

23) Miyata $T$, Horie $\mathrm{K}$, Ueda $\mathrm{Y}$, et al. Advanced glycation and lipoxidation of the peritoneal membrane: Respective roles of serum and peritoneal fluid reactive carbonyl compounds. Kidney Int 58: 425-435, 2000.

24) Akhand AA, Kato M, Suzuki H, et al. Carbonyl compounds cross-link cellular proteins and activate protein-tyrosine kinase p60c-Src. J Cell Biochem 72: 1-7, 1999.

25) Miyata $T$, Ishikawa $S$, Asahi K, et al. 2-Isopropylidenehydrazono-4oxo-thiazolidin-5ylacetanilide (OPB-9195) treatment inhibits the development of intimal thickening after balloon injury of rat carotid artery: Role of glycoxidation and lipoxidation reactions in vascular tissue damage. FEBS lett 445: 202-206, 1999.

26) Taguchi $T$, Sugiura M, Hamada $Y$, Miwa I. In vivo formation of a Schiff base of aminoguanidine with pyridoxal phosphate. Biochem Pharmacol 55: 1667-1671, 1998.

27) Miyata T, van Ypersele de Strihou C, Ueda Y, et al. Angiotensin II receptor antagonists and angiotensin converting enzyme inhibitors lower in vitro the formation of advanced glycation end products: Biochemical mechanisms. J Am Soc Nephrol 13: 2478-2487, 2002.

28) Nangaku M, Miyata T, Sada T, et al. Anti-hypertensive agents inhibit in vivo the formation of advanced glycation end products and improve renal damage in a type 2 diabetic nephropathy rat model. J Am Soc Nephrol 14: 1212-1222, 2003.

29) Michaelis OE, Patrick DH, Hansen CT, Canary JJ, Werner RM, Carswell N. Insulin-independent diabetes mellitus (type II) spontaneous hypertensive/NIH corpulent rat. Am J Pathol 123: 398-400, 1986.

30) Takaya K, Ogawa Y, Hiraoka J, et al. Nonsense mutation of leptin receptor in the obese spontaneously hypertensive Koletsky rat. Nat Genet 14: 130-131, 1996.

31) Parving HH, Hommel E, Jensen BR, Hansen HP. Long-term beneficial effect of ACE inhibition on diabetic nephropathy in normotensive type 1 diabetic patients. Kidney Int 60: 228-234, 2001.

32) Brenner BM, Cooper ME, de Zeeuw D, et al. RENAAL Study Investigators: Effects of losartan on renal and cardiovascular outcomes in patients with type 2 diabetes and nephropathy. N Engl J Med 345: 861-869, 2001.

33) Degenhardt TP, Alderson NL, Arrington DD, et al. Pyridoxamine inhibits early renal disease and dyslipidemia in the streptozotocindiabetic rat. Kidney Int 61: 939-950, 2002.

34) Soulis-Liparota T, Cooper M, Papazoglou D, Clarke B, Jerums G. Retardation by aminoguanidine of development of albuminuria, mesangial expansion, and tissue fluorescence in streptozocin-induced diabetic rat. Diabetes 40: 1328-1334, 1991.

35) Viberti G, Wheeldon NM. MicroAlbuminuria Reduction With VALsartan (MARVAL) Study Investigators: Microalbuminuria reduction with valsartan in patients with type 2 diabetes mellitus: a blood pressure-independent effect. Circulation 106: 672-678, 2000.

36) Heart outcomes prevention evaluation (HOPE) study investigators: Effects of ramipril on cardiovascular and microvascular outcomes in people with diabetes mellitus: Results of the HOPE study and MICROHOPE substudy. Lancet 355: 253-259, 2000.

37) Pitt B, Poole-Wilson PA, Segal R, et al. Effects of losartan compared with captopril on mortality in patients with symptomatic heart failure: Randomised trail-The losartan heart failure survival study ELITE II. Lancet 355: 1582-1587, 2000.

38) Weinberg MS, Weiberg AJ, Cord R, Zappe DH. The effect of high dose angiotensin II receptor blockade beyond maximal recommended doses in reducing urinary protein excretion. J Renin Angiotensin Aldosterone System 2 (suppl1): S196-S198, 2001.

39) Forbes JM, Cooper ME, Thallas V, et al. Reduction of the accumulation of advanced glycation end products by ACE inhibition in experimental diabetic nephropathy. Diabetes 51: 3274-3282, 2002.

40) Wilkinson-Berka JL, Kelly DJ, Koerner SM, et al. ALT-946 and aminoguanidine, inhibitors of advanced glycation, improve severe nephropathy in the diabetic transgenic (mREN-2) 27 rat. Diabetes 51: 3283-3289, 2002. 